\title{
Cycling around bends
}

\section{the effect of cycling speed on steering and gaze behavior}

Department of Movement and Sports Sciences, Ghent University, Belgium

\section{Introduction}

Although it is generally accepted that visual information guides steering, there is no consensus whether the tangent point strategy (the point of the inner lane boundary bearing the highest curvature in the $2 \mathrm{D}$ retinal image) or the gaze sampling strategy (looking at a points in the future path) is best suited to guide steering around bends. Unfortunately, visual behavior while negotiating curves has almost uniquely been tested in car driving situations and no effect of driving speed has been described yet. Therefore, current research investigates the effect of cycling speed on the visual behavior while cycling curves.

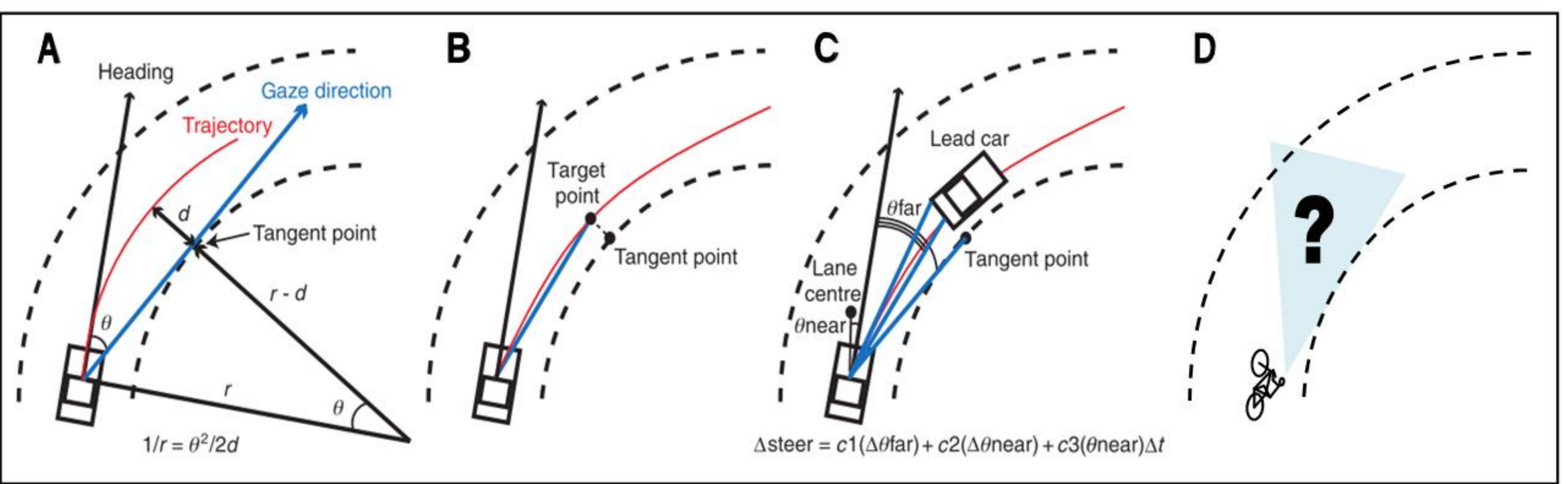

Figure 2 : Steering models for car driving (A-C) and cycling (D)

(A) Tangent point strategy according to Land and Lee (1994) (B) Gaze sampling strategy (Boer 1996; wilkie \& wann 2008) (C) Salvucci and Gray (2004) : The change in direction of any salient point along the road can be used to achieve proportional control of steering. (D) visual behavior while cycling trough curves has never been studied. (adapted from Mars 2008)

\section{Methods}

Figure 2 : Experimental set-up

1,s, Twenty-five participants (aged 21,83 $\pm 0,42$ y; 11females) were asked to cycle three times through a semicircle at three speeds : slow, preferred and fast. Seventeen participants with a

com Tracking Ratio $>90 \%$ were included in the analysis

Figure 3 : Participant wearing a head mounted eye tracker Gaze behavior was recorded using a $50 \mathrm{~Hz}$ Head-mounted Eye-tracking Device (SMI) which was mounted on a baseball cap and was connected to a notebook in a backpack.
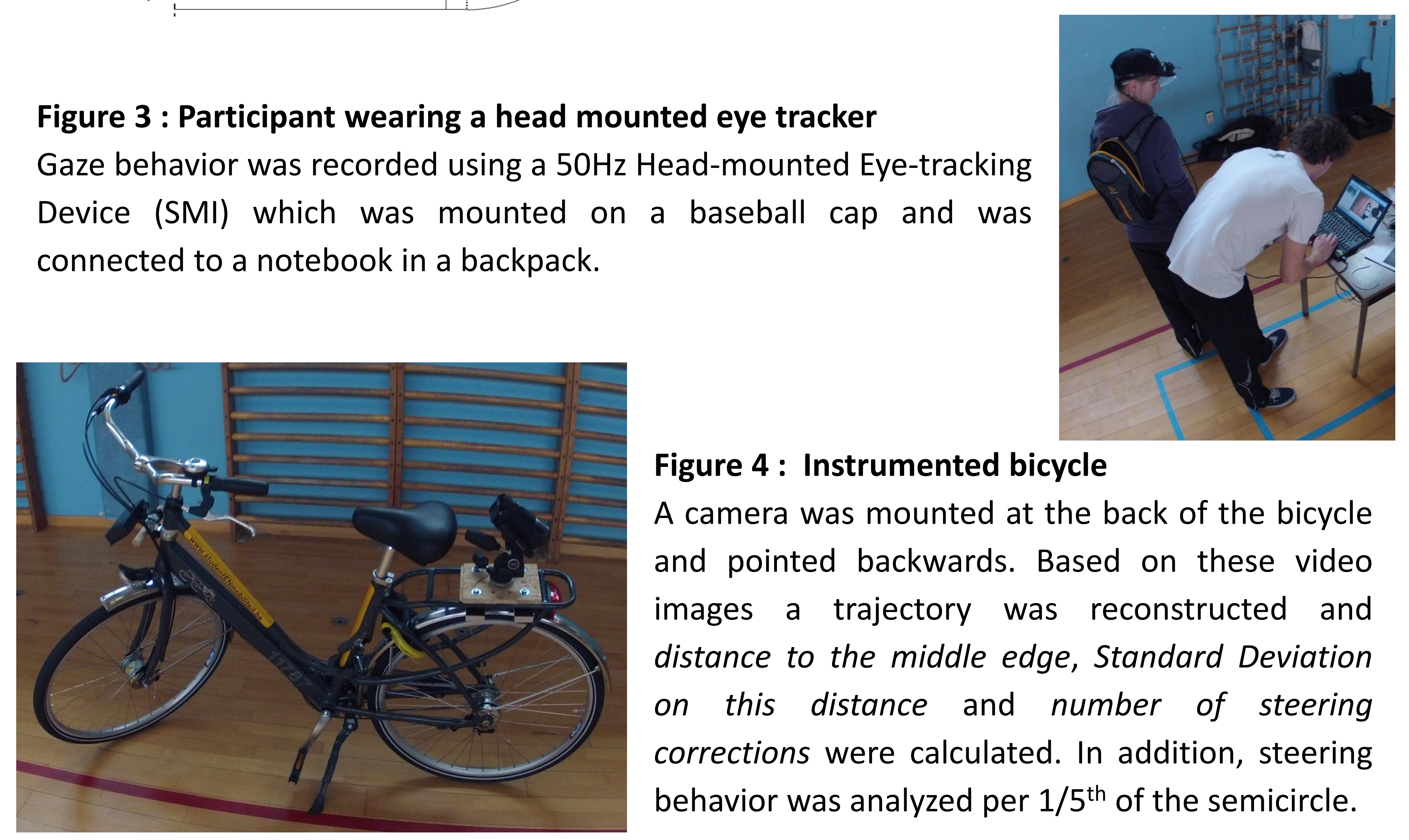

Figure 4 : Instrumented bicycle

A camera was mounted at the back of the bicycle and pointed backwards. Based on these video images a trajectory was reconstructed and distance to the middle edge, Standard Deviation on this distance and number of steering corrections were calculated. In addition, steering behavior was analyzed per $1 / 5^{\text {th }}$ of the semicircle.

\section{Results}

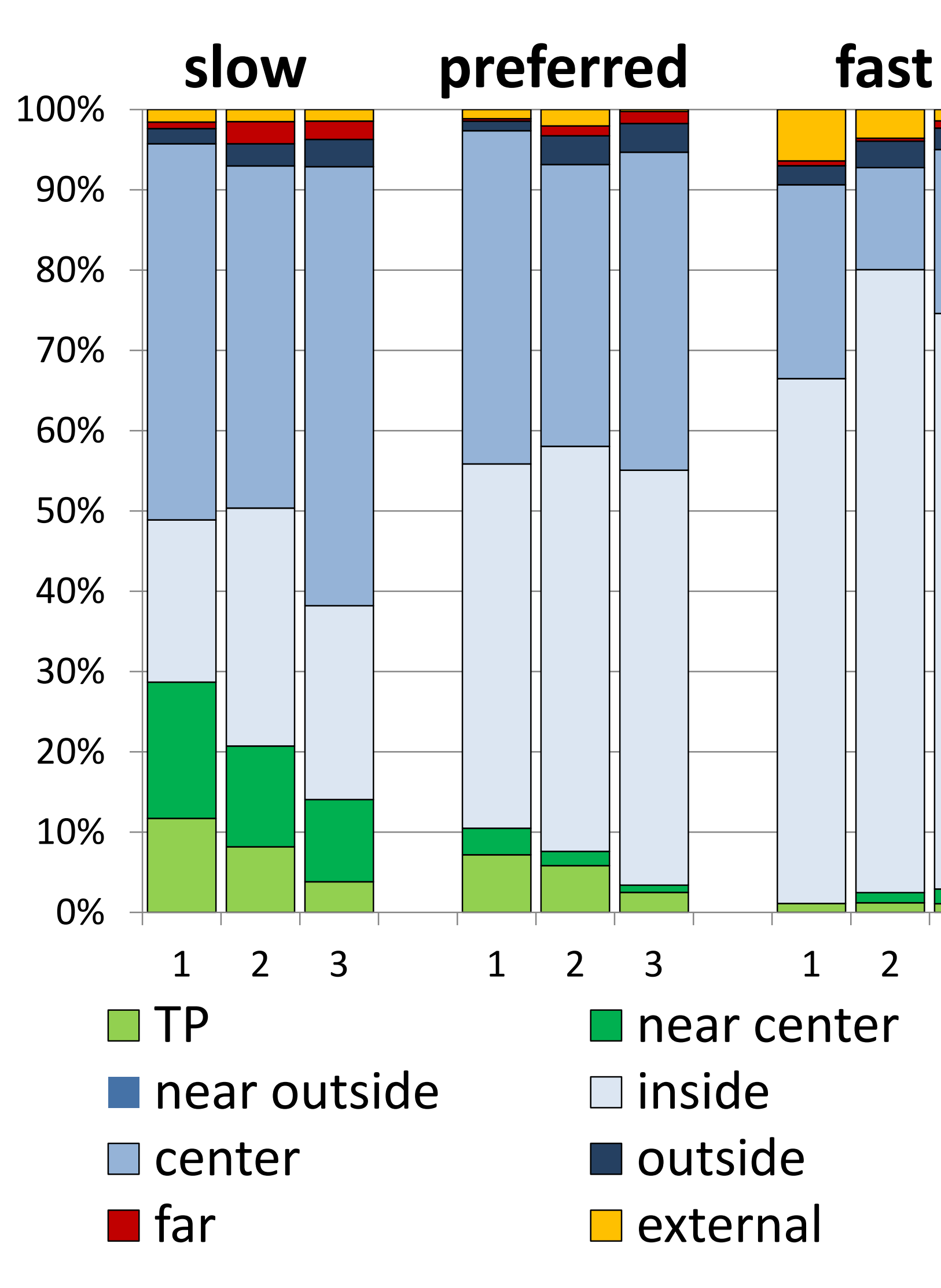

$\rightarrow$ Remark ! High standard deviations were found, indicating large differences in gaze behavior among the participants.
Corresponding author: pieter.vansteenkiste@ugent.be

Effect of increase of cycling speed and difference between 3 successive trials

Gaze
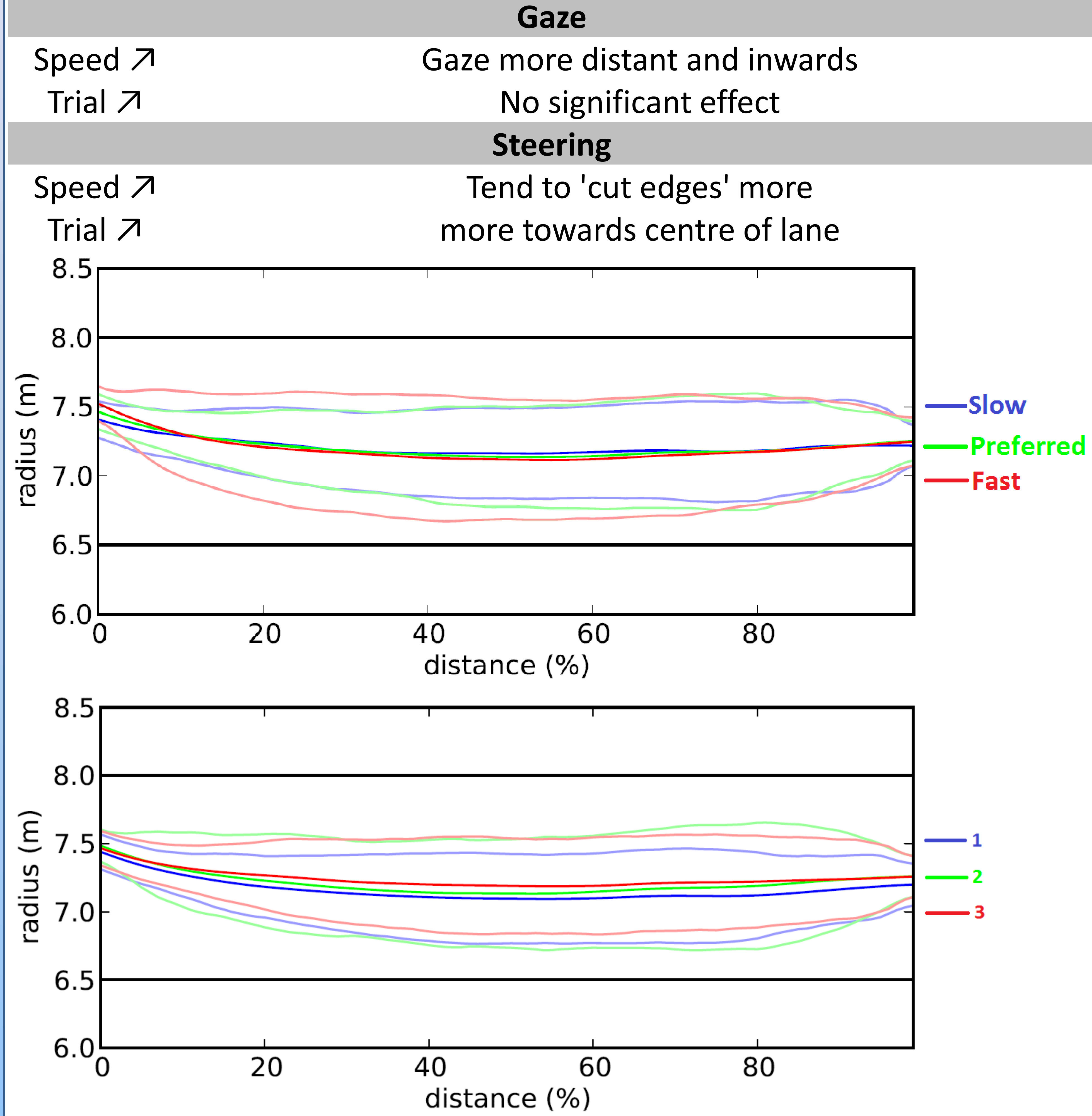

Figure 6 and 7 : Average cycling trajectory and standard deviation per speed and per trial Straight black lines represent edges of the cycling path. Light colors indicate standard deviation.

\section{Conclusions}

3angent point strategy was hardly used when cycling current curve

No proof for Gaze sampling strategy since gaze was often directed to inner edge

Gaze was strongly affected by cycling speed

DSteering behavior changed over trials, gaze behavior didn't

$\rightarrow$ steering is not only guided by vision but also by working memory

D Large variability in gaze behavior among participants

$\rightarrow$ Multiple visual strategies can be used for cycling a curve

\section{References}

Land, M. F., \& Lee, D. N. (1994). Where we look when we steer. Nature, 369, $742-744$

Salvucci, D. D., \& Gray, R. (2004). A two-point visual control model of steering. Perception, 33(10), 1233-1248.

Wilkie, R. M., Wann, J. P., \& Allison, R. S. (2008). Active gaze, visual look-ahead, and locomotor control. Journal of experimental psychology : Human perception and performance, 34(5), 1150-1164

Boer, E. R. (1996). Tangent point oriented curve negotiation. In Proceedings of the 1996 IEEE Intelligent Vehicles Symposium (pp. 7-12). Tokyo: Japan

Mars, F. (2008). Driving around bends with manipulated eye-steering coordination. Journal of Vision, 8(11), 1-11.

Vansteenkiste, P., Cardon, G., D’Hondt, E., Philippaerts, R., \& Lenoir, M. (2013). The visual control of bicycle steering: The effects of speed and path width. Accident Analysis \& Prevention, 51, 222-227.

\section{Acknowledgements}

This research was supported by the life line campaign of the Research Foundation of Flanders (FWO) FWO G.A115.11N. Special thanks go to Simon Depraetere and Thomas Roosen for helping with data collection and analysis. 